\title{
Epistemic Injustice in Research Evaluation: A Cultural Analysis of the Humanities and Physics in Estonia
}

\author{
Endla Lõhkivi, Katrin Velbaum, Jaana Eigi \\ Department of Philosophy, University of Tartu
}

This paper explores the issue of epistemic injustice in research evaluation. Through an analysis of the disciplinary cultures of physics and humanities, we attempt to identify some aims and values specific to the disciplinary areas. We suggest that credibility is at stake when the cultural values and goals of a discipline contradict those presupposed by official evaluation standards. Disciplines that are better aligned with the epistemic assumptions of evaluation standards appear to produce more "scientific" findings. To restore epistemic justice in research evaluation, we argue that the specificity of a discipline's epistemic aims, values, and cultural identities must be taken into account.

Keywords: epistemic injustice, research cultures, aims and values in research practice, evaluation

\section{Introduction}

The scientists interviewed during our study describe a state of affairs that supports Joseph Rouse's claim that the laboratory sciences "deploy more effective forms of power" (Rouse 1987, 202). ${ }^{1}$ As research evaluation standards

Corresponding author's address: Endla Lõhkivi, Department of Philosophy, Institute of Philosophy and Semiotics, University of Tartu, Ülikooli 18, Tartu 50090, Estonia. Email: endla.lohkivi@ut.ee.

1 The context of Rouse's claim is criticism on J. Habermas', C. Taylor's and R. Rorty's account of the humanities as purely interpretive and thus context-dependent sciences, whereas the natural sciences are seen by them, in one or another way, as a uniform kind, free of interpretation. Rouse finds this distinction to be inadequate and insists in his criticism upon the interpretive dimension of the natural sciences which has often been neglected. He explains the privileged position of the natural sciences by reference to power relations. The position is due not even to their strength in the laboratory or to having laboratories in the first place but to efficient use of laboratories as the instruments of power. The practices of the natural sciences include the ambition of manipulation and control of the research 
in Estonia have become increasingly bureaucratic, they rely on the quantitative publication and citation data collected in national electronic research information systems, such as ETIS, ${ }^{2}$ and the international Thomson Reuters Web of Science. Although such evaluation strategies are often presented simply as efficiency-oriented, and applicable to all academic disciplines, we argue that most are in fact compatible with laboratory sciences' standards and practices.

In this paper, we seek a cultural explanation why the laboratory sciences, such as physics, ${ }^{3}$ seem to have an advantage over humanities disciplines. In order to avoid repeating academic "folklore", or taking an a priori stance toward the scientific disciplines, we attempt to find out how scientists identify the position of their disciplines among other sciences and how they conceptualise their individual activities, pursuits, and careers in the academic hierarchy. At the same time, we try to retain a critical stance toward our empirical findings and interpretation. Our analysis is based on data from interviews with 36 Estonian physicists and 23 humanities researchers. Our method of analysis uses both culture contrast and the organisational theory of cognitive styles. Stephan Fuchs, a theoretical sociologist, contrasted the laboratory sciences with the humanities as the opposite ends of the entire spectrum of different scientific organisations and practices (Fuchs 1992), making a distinction that will be challenged by our empirical findings from both physics and humanities cultures. Theoretically inspired by the work of Alison Wylie (2011) and Miranda Fricker (1998, 2007), we use the concept of epistemic injustice to discuss the Estonian research evaluation model, because its criteria correspond to the interests of laboratory sciences better than the humanities. As a result, the latter fields suffer from unjust evaluation, losing their academic credibility. For the sake of epistemic justice we argue that cultural differences in disciplinary areas should be taken into account in their evaluations. A more just evaluation would prevent valuable contributions from being discounted or lost and would thus contribute to sustaining high quality of research. As a starting point, we will highlight several key characteristics and values specific to disciplinary practices throughout this paper.

In the following section (section 2) we address the issue of epistemic

objects-and laboratories are the best place for that-whereas the humanities do not have similar ambition. See (Rouse 1987, Ch. 6).

${ }^{2}$ ETIS-Eesti Teadusinfo Süsteem, the Estonian Research Information System, see www. etis.ee.

3 Physics, like many other academic research areas, involves both the elements of laboratory science and those of purely theoretical discipline, however, as we take theoretical deliberation to be part of research practice, and as it in the case of physics is directly or indirectly related to laboratory practices, we study physics as an example of laboratory science. 
injustice in the broader theoretical context of naturalised philosophy of science. Relying on Fricker's idea that in the conditions of competition the norms of credibility might start to imitate the structure of social power, we analyse the case of research evaluation criteria for the laboratory sciences and humanities in Estonia. In section 3, we describe our methodology for empirical investigation, the method of culture contrast-a qualitative method for cultural analysis which uses semi-structured in-depth interviews for gathering information about the culture being explored. In section 4 , a conceptual framework developed by Stephan Fuchs in organisational theory of scientific workplace cultures is analysed. Fuchs' characterisation of the scientific cultures in terms of mutual dependence and task uncertainty provides us with fruitful preliminary contrasts between laboratory sciences and humanities, but a closer look reveals that a more detailed cultural analysis is inevitable for the explanation of the power structure which appears via the evaluation results. Fuchs helps us to explain why the formalised scientometric evaluation is better suited for laboratory sciences in general, but since the disciplinary identities are no longer homogeneous in contemporary science, local cultural analysis of the research areas is needed.

The cultural analysis in section 5, illustrated by the interview quotes, brings the local values, aims and specificities of the disciplinary identities into light, for instance, showing radically different attitudes toward ideologies and the ways of arriving at the truth. The description of cultural differences enables us to suggest in section 6 what we find to be the reasons why humanities appear less credible based on the results of formal scientometric evaluation. The hidden epistemic assumptions of scientometric approach, the presupposed naïve empiricism, even though inadequate as an account for any discipline, any science, is better suited for the disciplinary cultures of laboratory sciences than humanities. The aims and values of the humanities appear to contradict the formalised scientometric model for several reasons-the humanities outcomes are not tracked by the information systems and indexes to a comparable degree with the laboratory sciences, short research article is not an approved genre of publication in the humanities, etc. We reach a conclusion that current academic evaluation standards mis-measure the achievements of the humanities, discrediting their disciplines in comparison to those in the laboratory sciences. Even if it is not intentional, it is epistemic injustice. For epistemic justice to be restored in research evaluation, the specificity of disciplinary aims, values, and cultural identities must be taken into account. 


\section{Theoretical considerations: the problem of epistemic injustice}

Until recently, academic analyses of scientific workplace cultures, identities, and styles of scientific work have been developed only in empirical disciplines such as science and technology studies (STS), sociology of science, and in some areas of gender studies and organisational studies. In the philosophy of science, the socio-cultural conditions of knowledge production have become a significant topic due to the rapid progress of social epistemology. Social epistemology involves, on the one hand, a normative philosophical approach that seeks to improve our understanding of the process of knowledge-making. On the other hand, the field relies on empirical data about the composition and dynamics of epistemic communities. The fastgrowing literature in the field shows why the study of research communities, specifically their organisation and culture, is relevant for the philosophy of science. For instance, Helen E. Longino has analysed what social conditions increase objectivity, suggesting a package of criteria for efficient transformative criticism (Longino 1990, 2002). Her model of transformative criticism is meant to facilitate communication between researchers with different background assumptions and social positions, so that all competent community members can participate in the dialogue. Ideally, this communicative model would enable researchers to detect and remove biases and mistakes and thus lead to more objective research results. But to remove communication barriers, one has to be able to recognise them. Gender biases can be one of those: Kristina Rolin has argued that gender is a relevant issue for the philosophy of science since it may influence a researcher's credibility in a particular community. In other words, because of the influence of certain power structures (e.g., gender) on communication practices, members of underrepresented groups may experience epistemic injustice, and objectivity criteria may be neglected (Rolin 2004). The epistemic integrity of a research group, or larger research community, depends to a great extent on the efficiency and character of their communication. In this paper we will understand the natural sciences, like any other field of research, as cultural groups characterised by certain power structures that have direct influence on their communication practices. The issue of epistemic injustice is the focal point of Alison Wylie's recent paper, in which she analyses the standpoint theory of feminist epistemology with respect to the issue of social segregation in the sciences. ${ }^{4}$ She defines epistemic injustice as a misrecognition of subdominant knowers and subdominant forms of knowledge (Wylie 2011, 162). Referring to Miranda

${ }^{4}$ In her paper, Wylie shows that the empirical data collected by feminist activists about the large number of minor cultural obstacles for women and/or racial minorities in the everyday workplace practices in science institutions, serves as a proof for the claim of hermeneutic and testimonial injustice. 
Fricker's work $(1998,2007)$, Wylie describes this process in terms of testimonial injustice, calling it "a form of systematic epistemic misrecognition", arising when "norms of credibility 'imitate structures of social power', so that 'working indicators' of rational authority pick out the powerful and not necessarily the knowledgeable or the truthful" (Wylie 2011, 160). Wylie refers to another type of misrecognition, termed 'hermeneutical injustice' by Fricker. This form of misrecognition indicates "systematic gaps in the interpretive resources available to epistemic agents that put those who are marginal socially and materially at an epistemic disadvantage, not just testimonially but also conceptually and communicatively" (Wylie 2011, 161). Wylie points out that testimonial injustice goes along with a loss in credibility and thus misrecognition of epistemic agents, which in turn results in institutional inequity concerning hiring, tenuring, promoting, and awarding grants.

According to Fricker, the norm of credibility is a fundamental norm of any epistemic practice (Fricker 1998, 172). In the conditions that she describes as "minimal practice in the state of nature", this norm is operating reliably, its indicators pointing to rational authority. However, under the social pressure, for instance, in the conditions of competition, the norm of credibility might start to imitate the structures of social power, and its workingindicator-properties will then tend to pick out the powerful as more credible than powerless. In these circumstances, while one group experiences a credibility deficit, the other experiences overspill. Biased credibility norms, as numerous empirical studies on gendered workplace cultures have shown, ${ }^{5}$ and as Wylie indicates, translate into patterns of hermeneutic injustice, rather than open and deliberate discrimination. An unfriendly working environment, unfair local distribution of smaller tasks between researchers, and hostile everyday communication patterns can culminate in hermeneutic injustice, testimonial injustice, and a loss of credibility. Furthermore, it is possible that a chilly workplace climate and gender segregation will impact the overall development of a field of study; topics and methods will likely be selected according to dominant viewpoints, and decisions are likely to be made by dominant groups.

In our case study on Estonia's cultures of physics and the humanities, we apply the theoretical notion of epistemic injustice not to race, age, or gender, but to the cultures of research disciplines. Application policies for funding and their respective evaluation procedures have been discussed in public, and are therefore deemed to be fair and transparent. All disciplinary areas have been declared to be in equal standing in grant competitions, with quality and efficiency being the major determining factors for all. Nevertheless, a

5 See, e.g., (Rossiter 1995), (Hasse and Trentemøller 2008), (Hasse and Trentemøller 2011),(Wennerås and Wold 2001). 
closer look at local research cultures in the conditions of competition reveals both hermeneutic and testimonial injustice, as our empirical study shows. The competition for resources has its influence on the operating credibility norms. The positioning of disciplinary cultures in the academic landscape seems to display the traits of epistemic injustice with respect to the evaluation criteria in the humanities in comparison to the natural sciences. More specifically, the humanities experience difficulties with grant and project applications due to the evaluation standards in Estonia. ${ }^{6}$ According to the current regulations, the evaluation is based to a large extent on such quality criteria as the researchers' $h$-index, number of citations, previous success in obtaining additional funding, participation in the international projects, doctoral schools, etc. ${ }^{7}$ Hermeneutic injustice, in this case, results from a lack of understanding of the differences in disciplinary cultures and their everyday practices. For instance, the impact factor, $h$-index, is not applicable to the humanities in the same way as in the natural sciences simply because most of the research outcomes, either academic journal articles or monographs, in this area are not indexed by the Web of Science. Evaluation based on $h$-index makes the humanities in general seem far less trustworthy than they deserve. Secondly, the fact that citation practices differ significantly in the humanities, social sciences and natural sciences, has not been taken into consideration. Internationally, the variation of citation practices disciplinewise has been analysed, e.g. by Audrey Baneyx (2008), Nancy Adler and Anne-Wil Harzing (2009). These authors claim that in comparison to the Thomson Reuters Web of Science, Google Scholar and other new information systems take more humanities and social sciences publications into account and provide a more balanced view.

One of the central theoretical principles for naturalised philosophy of science is the need for the scientific success to be assessed in terms of the degree to which the scientists have achieved their aims and goals. ${ }^{8}$ The evaluation criteria should not contradict researchers' values and aims. Currently the specific aims of research in the humanities, the researchers' selfperceived identities (i.e. their role both in academia and in wider society), the local approach, and the method-specific features of the workplace are ignored by the Estonia's academic grant and project application evaluation standards. As the empirical interview material shows, researchers in the hu-

${ }^{6}$ Since nearly all research funding in Estonia is project based, the extent and quality of the research activities depends entirely on the success of the project applications. For further information see the website of the Estonian Research Agency: www.etag.ee.

7 For more information, see "Guidelines for evaluation institutional research funding applications", www.etag.ee/wp-content/uploads/2012/05/ IUT-taotluste-hindamisjuhendi-kinnitamine-LISA.pdf.

8 E.g., (Giere 1988, 7). 
manities are not against evaluation, but the evaluation should concern more the very content and quality of research, rather than formal indicators, such as the number of citations, publishers' ranking, etc. The analysis of the epistemic background assumptions of the established evaluation criteria shows that the general form of inquiry of the laboratory sciences serves as the epistemic model for universal evaluation criteria. ${ }^{9}$ Because they are assessed based on the criteria of the laboratory sciences, the humanities lose credibility, have less epistemic authority, and are therefore less able to define what counts as good research.

\section{Sources of Empirical Data and the Culture Contrast Method}

In this paper, we seek a local cultural explanation for the epistemic inequity, asking what factors make the existing evaluation criteria unjust for the humanities. Our analysis relies on the empirical data collected in the course of an international research project aiming at a cultural explanation for a recent phenomenon that many researchers have left academic careers in physics. On several occasions during the investigation of physics cultures, questions about humanities cultures arose. In order to address them, we extended the method of culture contrast, which was already in use for the empirical study of physics culture, to a study of the humanities. Alongside our empirical investigation, we discuss, in philosophical terms, what practices scientists see as epistemically preferable for conducting high-quality research, and why.

In the interdisciplinary project called UPGEM (Understanding Puzzles in the Gendered European Map Brain Drain in Physics through the Cultural Looking Glass) spanning the years from 2005 to 2008, we studied physics workplace cultures with partners from five European countries. We paid special attention to scientists' career choices and identity formation, seeking gendered patterns of inclusion and exclusion of people, ideas, and work styles (Velbaum et al. 2008, Hasse and Trentemøller 2008, see also www. upgem.dk). Our subsequent project was initiated in Estonia in 2010, and aims to explore the cultural similarities and differences between the physical sciences and humanities. During the pilot period, we did not select one particular disciplinary culture for investigation, but instead addressed the humanities as a whole. Since 2011, our focus has become narrower, and we specified history as our main field of research in the humanities. In the current paper, however, we use all the material collected so far and address humanities in general.

59 semi-structured in-depth interviews were made ( 36 with physicists and 23 with humanities researchers). Roughly half of the respondents were

\footnotetext{
9 See section 6 in this paper.
} 
male and the other half were female. There were also interviews with those who used to work either as physicists or humanities researchers, but had left the field by the time of interview. The scope of respondents according to academic career level was chosen purposely wide-ranging from $\mathrm{PhD}$ students to full time professors.

Data was qualitatively analysed using the culture contrast method that enables making visible the context in which we act, the cultural factors that guide us, in order to understand "what makes people think-feel-talk-act in ways that everyone in their group takes to be normal" (Traweek 1992, 440). The empirical material was treated with care and respect but quotations were not perceived as mechanical building blocks of current analysis. It is taken into account that their final meaning is established in comparison with the other interviews used here.

Overall, the method can be expressed by three questions:

1. What effects do researcher's categorisations have on informant's categorisations?

2. What effects do informant's categorisations have on researcher's categorisations?

3. What effects do the researcher's categorisations have on other researchers' categorisations? (Hasse and Trentemøller 2009, 53)

The method of culture contrast focuses mainly on the second type of questions: when science, an academic culture, becomes the object of research, many categories are used in a similar way by the researcher and the interviewee. But the use of certain key words and ideas can vary between different scientific disciplines, making it necessary that the interviewer be open to the dynamic development of research categories during the interview. The expansion of the semantic field of the categories ensues from allowing for one's prejudices to be challenged.

Using the method of culture contrast, we attempt to identify and analyse what disciplinary values emerge as culturally-specific. We proceed with the assumption that the cultures (and practices) of the natural sciences and humanities differ. Though the disciplines share some similarities, i.e. both are regulated by the unified Estonian science-policy system, the local meanings of many conceptual categories and research methods remain different. The humanities were studied after physics cultures, resulting in an analysis heavily influenced by the latter's cultural categories. In other words, the meanings of physics culture became presuppositions. When similar questions were applied to humanities cultures, contrasts emerged. 
The quotes in the paper have been selected from among several similar ones to give evidence of the existence of certain views, positions and attitudes. They have been compared with each other and those which express the point most clearly or strongly have been cited by us.

\section{The Workplace Cultures of Physics and Humanities}

In this section we are going to characterise the types of work organisation in the humanities and physics research. In order to achieve the epistemic aims of a group, research work has to be arranged in an efficient way in the workplace. The types of organisation as well as the local cultures vary from one discipline to another. The analysis of organisation patterns and the local cultures and practices enables us to reach better understanding of the roots and development of the epistemic injustice in evaluation.

When we began our comparative analysis of physics and the humanities, we assumed that the two disciplinary areas were distinctive cultures and would thus provide us with suitable material for the method of culture contrast. We chose Stephan Fuchs' distinction of cognitive styles in science as a general framework (Fuchs 1992), with the intention that it would bring to light and explain certain anticipated differences. Fuchs uses a sociological lens to examine the work styles, form of knowledge produced, and the similarities and differences between various sciences and humanities disciplines. His theory uses two key concepts of organisational theory: mutual dependence and task uncertainty. Mutual dependence describes the extent to which scientists' work depends on the work of other scientists and other institutions that distribute resources and professional accreditation. High mutual dependence results in uniform and universally-accepted research practices; as control over resources is concentrated and centralised, alternative and idiosyncratic approaches are not supported. Low mutual dependence allows for the coexistence of multiple approaches and paradigms; accordingly, less uniformity and agreement exists about methods and results. Task uncertainty describes the extent to which scientific production is routinised and predictable. Uncertainty is low when problem definitions, problem-solving methods and criteria for judging solutions are stable and universally agreed upon. Uncertainty is high when problems are not clearly defined, and methods and criteria are multiple and controversial.

According to Fuchs, the characteristics of high mutual dependence and low task uncertainty are typical for hard natural sciences. These are expensive areas of research and require a great deal of highly concentrated resources. Statements that are supported by such resources, and are regularly cited by other scientists, are unlikely to be questioned. Accordingly, the natural sciences are oriented toward producing solid "facts", requiring close 
cooperation between both individuals and groups. Scientists are therefore usually confident about the validity and rationality of their research methods and the knowledge they produce. Our interview material confirms this characterisation:

Interviewer: The next question goes well with what you just said, in your opinion, is pursuing physics individual or group work in its essence?

$\mathrm{P}_{320}:^{10}$ It should be more about group work, yes, for there to be any results. Otherwise, there's just so much work that if you do it all by yourself, you won't get anywhere.

Interviewer: Do you consider the work of a physicist an individual thing or is it a group effort?

$\mathrm{P}_{333}$ : It's both. It's strictly a group effort in the sense that a selfemployed person can be a tailor, a hairdresser, a beautician, but a physicist can never be a self-employed person. So you won't be able to sell formulae on the market [laughter] and what a physicist does acquires a meaning in relation with, in connection with the science of physics that is done by a large number of people. So this kind of a [...] financially nor content-wise can an independent physicist do anything independently.

In the contemporary context, scientists have expressed frustration, arguing for a change in science policy that would stop the dispersal of resources and concentrate them in the hands of those groups that deal with the most important and/or prestigious problems in the field, and efficiently produce innovative research. For instance, one Estonian physicist expressed his disappointment:

P 302: Money is divided equally to all considering the personalities, not according to what they do or what they're capable of. Science is an elite area, some can handle it and some can't. The ones that can, should get the money.

Another physicist resonates with the worry about the loss of time and other resources caused by the project-based organisation of work:

P306: The financing of science used to be like a clock-work, that the scientists were freed from managing the laboratory. Now they have to if they want to do something more serious, think in economic terms

${ }^{10}$ Capital letter ' $\mathrm{P}$ ' refers to interviewed physicists and, respectively, ' $\mathrm{H}$ ' refers to humanities researchers. The interviews have been transcribed one-to-one without any linguistic correction or abbreviation. The individual way of expression has been preserved also in the English translation. 
and plan the activities. ... [S]cientist has to be now an entrepreneur and I don't like it. ${ }^{11}$

In contrast, low mutual dependence and high task uncertainty are typical for the social sciences and humanities; resources are less expensive and more widely dispersed, allowing for multiple schools and traditions. Social sciences and humanities statements are less likely to turn into universallyaccepted "facts"; instead, these fields produce "conversations" and hermeneutics. There is more space for disagreement, alternative interpretations, and less pressure to cooperate. A critical and self-reflexive attitude toward both methods and results is common and desirable.

Fuchs suggests that certain fields become highly bureaucratised when research follows standard methodological rules, because findings can be assessed using formalistic criteria and colleagues can routinely measure the value of each other's contributions. High bureaucratisation is only possible with low task uncertainty, which produces relatively uniform research and evaluation practices. In turn, these features are typical of organisations with high mutual dependence and cohesion, those of the natural sciences. On the other hand, high uncertainty and a lack of clear problem definitions contradict bureaucratisation; these qualities produce a variety of methods, a plurality of local standards, and possibly disagreement over findings and their merit. Pluralism and disagreement are typical for fields with low mutual dependence, e.g., the humanities. This difference between the two cultures was recognised by our informants. One physicist described the difference in terms of method:

$\mathrm{P}_{333}$ : Well, first and foremost physics differs from humanities because it has a certain method. So whereas the humanities, let's say, psychology, sociology, could be compared to a bush where there are different schools, right, different schools follow from different assumptions, and a very strong thing is quotation and authority. ${ }^{12}$

Fuchs' distinction explains why bureaucratised control and evaluation is associated with, and suited to, the natural sciences. Compared to the humanities, the natural sciences have lower task uncertainty and higher mutual dependence, which are the prerequisites of formal, rigid, and bureaucratised

${ }^{11}$ These two quotes need to be seen in the historical perspective of the Soviet-time large and relatively efficient research institutes where the academic personnel was free from administrative and economic concerns. The first interviewee has adopted the new high research standards and favours a competitive pattern of distribution of resources, whereas the other insists on the nostalgic image of time of research in conditions of unlimited resources (Velbaum et al. 2008).

${ }^{12}$ By the quotation and authority the informant means here not the quantitative indicators but the traditions that in humanities are often related to renowned scholars and fashionable trends or ideologies. 
evaluation procedures. So, if we use Fuchs' distinction as our point of departure, it is plausible to suggest that as Estonia's current evaluation procedures are increasingly formalised, more of a negative response would be provoked from the humanities than from the natural sciences. Yet our research has shown that reactions to evaluation procedures, and the topic of evaluating research quality more generally, are more nuanced and complex both in the humanities and the natural sciences. The first point that counters Fuchs' picture of the two opposites is our observation that both the natural sciences and the humanities suffer from uniform bureaucratic evaluation procedures. Many bureaucratic requirements and standards run contrary to the policymakers' intentions, resulting in diminished efficiency and quality. Further, bureaucratic restrictions can stifle innovative ideas and critical dialogue in the scientific community:

$\mathrm{P}_{318}$ : Now there are a hundred thousand officials who you constantly have to hand in reports, lie all the time. They make people lie all the time. [...] [T] he mediocre ones sit and can't do that much science but they feel comfortable being science managers-they dabble a bit in a laboratory and then go to a meeting where they start ordering others around...

In addition, exaggerated bureaucracy may result in the exclusion of significant critical perspectives. What seems even more worrisome is that researchers have started to plan their activities to produce results that best match fixed bureaucratic categories, creating projects that are designed to produce as many high-rank publications as possible. This indicates that the hierarchy of the publication categories of the research information system is currently providing direction to the activities of some researchers. This is so both in physics and the humanities, but as we shall see in the following sections, the pattern contradicts the disciplinary orientation and aims of the humanities more than physics. An informant in the humanities has characterised the difficulties of planning the unit's activities in the conditions of the internal conflict between real research interests and the power of the academic information system as follows:

Ho2: It is not set, nobody gives orders, but we have the Research Information System, which is not an information system but the need as a head specialist to be a leading scientist [...] I mean that one's research should be chosen in the interests of what is-what is the specialty's material future, not only in terms of content. I mean, one should aim at research that is financed, and my specialty is [...] I mean it does not belong in the category of small specialties, but it still is a small specialty. It is national literature, it should be highly respected, but my task is to make it international. So that those priorities I need to determine myself in my research, have to decide myself, are actually 
to a large extent compromises between what I am really interested in and what is required in institutional terms so that my specialty would get financed.

Second, according to Fuchs' preliminary contrast, the natural sciences are seen as producing solid "facts" whereas the humanities and social sciences are interpretive and produce "conversations". This distinction can be challenged by two arguments:

1) As with many other academic disciplines, the natural sciences, including physics, should be seen as a narrative-enacting practice ${ }^{13}$ in which the emerging "facts" are a part of the activity which produces conversations specific to physics. To put it more precisely: solid facts are produced by conversations within the field of physics. Similarly, humanities' conversations produce not only interpretations, but some facts (e.g., historical, ethnographic, anthropological, archaeological) as well.

2) The nature and organisation of research varies from one research group to another within a discipline, and local workplace cultures differ in aspects other than mutual dependence and task uncertainty. Different people, ideas, and practices are included and excluded, the local power structure and the level of epistemic (in)justice varies from one place to another. Therefore, a more detailed analysis of the local cultural context of the natural sciences and humanities is necessary. Our cultural analysis is inspired by Joseph Rouse's concept of heterogeneous alignments:

Epistemically significant groups are defined not by the common possession of some presupposed content but by their belonging to partially shared situations, to a cultural field. "Science" and scientific knowledge are not framed by the identification of scientific communities but are among the meanings at issue in the formation and interaction of various cultural alignments and groups. (Rouse 1996, 112)

Based on this idea of cultural fields and partially shared situations, we explore how cultural alignments emerge and are sustained both in the humanities and the natural sciences. We take the natural sciences and humanities not as fixed disciplinary areas with static internal features, but rather as culturally created fields of practices.

Because the method of culture contrast presupposes the possibility that assumptions will be changed during the course of study, we were prepared

${ }^{13}$ Rouse explains the narrative enactment as follows: "Scientists make sense of what they are doing by understanding it as a response to the situation presented by past research and anticipation of future developments. That is, they continually enact a narrative in the midst of which their present activities (and those of others) would be intelligibly situated" (Rouse 1996, 27). 
to modify our initial categories if necessary. Accordingly, we modified and expanded both our initial categories and our interpretation of Fuchs' ideas, combining them with additional frameworks. In the following section, the interviewed scientists' identities will be analysed. As Sharon Traweek's study of the high energy physics communities in the US, Japan and Europe has shown, professional identities are created and modified during the entire period of training and subsequently, throughout one's research career (Traweek 1992). Identities may vary from one career level to another, different individual skills and talents are evaluated by novices and advanced researchers, and different temperaments may be required for recognition in a particular research group. Organisational characteristics of a workplace culture, like those identified by Fuchs, in combination with the following analysis of disciplinary identities, will provide us with a "map" of the aims and values present both in physics and humanities cultures. The description of these identities and values will then be compared with the presuppositions of science policy instruments, such as evaluation criteria. This comparison will allow us to suggest some sources of epistemic injustice.

\section{Disciplinary Identities, Aims and Values}

Following Traweek's anthropological approach, we see disciplinary identities not as predetermined by shared theoretical consensus or a given list of values, but instead as something developed and modified by an epistemic group, based on a shared history and future perspectives. "Allies" and "enemies" are formed in response to encountered situations, people, and problems to be solved. This approach has been endorsed by Rouse, who contrasted the post-positivist (consensus) philosophy of science with the cultural studies' perspective, giving credit to the latter for addressing "the ways in which meaningful differences and boundaries are established, sustained and transformed; the ways they fragment; and the tensions and resonances which indicate that apparently stable identities and differences are sustained only by ongoing work" (Rouse 1996, 111).

In our analysis of the collected interview material, we focused on the following questions:

1. how do these scientists see their disciplinary and personal position in academia and in society more generally?

2. how do scientists define the aim of their research work?

3. what is the basis for evaluating others' work?

4. what kind of obstacles do scientists identify in their work? 
We critically reflected on the interview material by contrasting the views of our informants with each other and our own presuppositions. Our starting point was the new categories that emerged from the interviews with humanities researchers as compared with the physicists' interviews. These categories concerned humanities researchers' identity, goals and values. Although not universal, their presence was strong enough to merit analysis. We found that like physicists, humanities researchers had two focuses for articulating the meaning behind their activities: the general goals of their research, and their chosen methods.

The physicists stated that their goal is to explain and understand fundamental processes of the world through simplification and idealisation, whereas humanities researchers cited their concern with the concept and meaning of being human. In other words, humanities scholarship is related to questions that address the identity of a person, group, or nation. In the interviews, the humanities researchers presented the idea that being human is strongly correlated to the knowledge and awareness of one's cultural identity and context; an understanding of one's national history and culture contributes to one's humanity and level of maturity. In fact, ignorance about one's culture was said to create an impression of infantilism. The humanities notion of what defined an adult identity stood in opposition to the identity of the "playful boy" that emerged in our interviews with the physicists. The latter group described the value of a childlike joy of discovery and the ability to hold onto one's naïve curiosity.

This contrast manifested itself in two ways. First, it was connected to the notion of creativity, which was deemed vital to both physicists as well as humanities researchers. Physicists defined creativity as the ability to solve problems. The "territory" of creativity was broader for humanities researchers, who saw creative self-expression, through the production of art or literature, for instance, as one of an individual's main competencies. ${ }^{14}$ Creative output that is valued in humanities is not limited by scientific publications. In response to the question of why they might quote a colleague's work, one humanities researcher said:

Ho1: [A]nd [...] while I absolutely don't care about their some kind of index or some status. If I see that that any writer thinks interestingly, asks the right questions, it is not necessarily important that they have been published by an academic publishing house or in an academic journal.

In physics, creativity is mainly seen as a problem-solving activity, but also as playfulness:

${ }^{14}$ For an international comparison, see Hemlin and Gustafsson (1996); in their quantitative study they also showed that in the humanities the individual talent is a dominant value. 
P306: What I have admired most is that people are competent in their discipline, they orientate freely in it, are able to think freely, smell of sweat disappears from this whole thinking process. It's like a game where you can think one way or another.

So, in discussing creativity physicists do not talk about extending the areas of its application but rather about particular forms of realising creativity in the primary area of their work.

Second, this contrasting view of the characteristics of an "adult" was relevant to the researchers' attitudes toward themselves and their age. While physicists maintained the more common opinion that the most productive creative period for "real" discoveries is youth, the humanities researchers believed that the quality of one's endeavours improved with age. They described expertise as a characteristic that requires time to mature. However, they argued that it was vital to acquire the skills and attitudes in youth that cannot be attained later in life. These skills and attitudes relate to research methods and can be described under the umbrella term of "criticism". Humanities researchers believed in the significance of self-criticism, or the willingness to review and re-review one's existing points of view, sources, and theories.

Hog: Being an historian means to be able to adequately evaluate the sources you are working with, ask questions about the research object, and then set goals, one has to be very consistent, to achieve the goals and answer the questions posed at the outset.

Only after acquiring the critical method, the complex of competences and skills for working critically with the primary sources, the existing literature, methods and perspectives, one can qualify as a historian. It is this set of skills that distinguishes a professional historian from an amateur that approaches research material uncritically and without reflection upon one's methods.

According to the interviewees, one's ability to acquire these skills depends on one's training, bringing us to another important contrast between the two disciplinary areas with regard to teaching. The physicists rarely mentioned teaching as a part of research work; in several cases teaching seemed to have a negative connotation for them, and was described in terms of the opposition "the scientist versus the teaching scholar". In this relationship, whereas the scientist is a producer of new knowledge, the scholar/teacher is a transmitter of ready-made knowledge. In the humanities, the interviewees presented a more positive attitude related to teaching, as an opportunity to test one's ideas:

Ho1: In general, I like lecturing, [...] and often I get good feedback from the company in the classroom, it enables me to order my ideas and think through my areas of investigation. 
In addition, the idea of responsibility toward younger generations was expressed more prominently by the researchers interviewed from the humanities.

Another contrast emerged with respect to the pursuit of "truth", for which the methods and goals of physicists and humanities researchers differed. In the interviews with the physicists, we identified a perception of the researcher as the "priest of truth": a person who serves only the truth, and aims at uncovering an objective picture of nature. Thus, physics was depicted as an area free of ideology:

P309: I am in science strongly convinced that what is called the faith in truth or serving the truth, or in other words, in order to achieve results in science one has to [...] well, the objective truth, as such [...] as such an ultimate goal is placed in the most important position. And other values would largely have to be subjected to that...

Serving the truth is supposed to take precedence over other values like personal career advancement but also over other goals science may be made serve. A physicist emphasised that the natural sciences have historically been free of ideological pressure, even when operating within a totalitarian society. Their ideological neutrality is contrasted starkly with the state of humanities.

P301: I was interested in very many things, but the main reason why I and many others started with science subjects was that it was a relatively honest job. It was an area where there wasn't any ideological pressure, none whatsoever. Completely honest areas.

Interviewer: What kind of ideological pressure? You mean compared with humanitarian areas?

P301: Yes, compared to humanitarian areas where it was totally crazy.

The researchers in the humanities were aware that those in the sciences saw humanities research entirely as ideology.

Ho9: So, I had the conversation with the [physicists] [...] It was interesting, the topic of conversation was whether the art history is a science. Eventually they accepted that it can be a science, whereas history as such cannot, in no way. For them it is only such an ideology.

Humanities researchers recognise this perception of humanities as ideologyladen. Unlike physicists, they do not attempt to present good research as ideology-neutral. They readily admit that humanities research always includes a broader ideological dimension. Pursuit of truth in humanities relies on its interpretation and critical analysis rather than an attempt to dismiss it from the picture: 
Ho4: [...] [N] ational science has been in some occasions thoroughly ideology-laden in Estonia. And it is being thought that it always is. [...] [A]ctually since 1990 os our [discipline] has been very critical.

Truth is revealed only through critical analysis.

During the interviews, we also addressed the criteria informants used to assess the quality of their own work as well as that of their peers. Their answers revealed specific disciplinary values. As it was mentioned earlier, physicists value highly the ability to solve problems, and it was regarded as the main qualification and research competency:

P319: We must have competence. And precisely-and physicists are important in this sense, that they are accustomed to solve unexpected situations, non-standard tasks.

For humanities researchers, value was placed on the ability to maintain a productive and positive relationship with issues relevant to the local culture. A vigorously self-reflective perspective was also necessary:

Ho3: [T] he humanities are still those that reflect national culture. [...] [L]et's say even in literature it is so that literary scholars don't have to write books themselves but they definitely have to say something about those books that exist in the literature that they study, so reflection is still important also there.

A formal criterion for humanities researchers is producing written works that are thorough and in monograph format. For humanities researchers to produce worthwhile analyses and criticisms of ideological frameworks, they must undertake an extensive historical and historiographical study of the problem, as well as a self-critical reflection on one's methodology and choice of topic. Attention to the quality of one's writing is also considered important-it is valued when humanities research is presented in the best possible language. However, current science policy discourages writing monographs, particularly in case of emerging researchers:

Ho2: I can't encourage any doctoral students to publish a monographic dissertation. [...] Everyone is defending using the article-model because otherwise, if they don't get a PhD then research institution can't register them so that they get paid. This is the project-based management.

A younger-generation historian described the conflict between the expectations of wider society, and those of academic culture. The latter pushes historians to publish shorter expert articles in academic journals that are inaccessible to the public: 
H12: A historian does not write monographs today anymore, and as $s$ /he does not write monographs, it is practically impossible that somebody just interested in history could read something, it is impossible for a layperson to read scientific journals.

Journal articles may be adequate for sustaining communication between professional historians but they are not meant for lay readers. Accordingly, historians can no longer address lay audience and thus play an immediate role in wider societal cultural debate.

In this interview, and in many others, a conflict emerged between the values and aims researchers see for themselves in connection with the broader social and cultural context and those imposed on them by science policy regulations. Humanities researchers seem to be suffering more from this value conflict than physicists, as we explain in the following section.

\section{Epistemic Injustice in Disciplinary Landscape}

In science policy decisions, especially those concerning allocation of resources, the promotion of one research field over another, or the distribution of general tasks, an evaluation of previous results and an analysis of the available capacities and resources is required. In this section we shall see how the hidden epistemic assumptions operate in the evaluation practices. Any evaluation model involves specific epistemic assumptions: the description of the ideal situation of knowledge production, the ideal structure of scientific progress. To a great extent, evaluations of grant and project applications are based on scientometric data and analysis. Daryl Chubin and Sal Restivo characterise the epistemic assumptions and the underlying metaphysics of the scientometric account of science as rather simplistic:

For the scientometrician the world exists sui generis; it can be sampled, measured, captured, and revealed according to 'objective inquirers'. Those inquirers 'make estimates' and 'test hypotheses' rather than 'make knowledge claims' and 'construct realities'. (Chubin and Restivo 1983, 57)

They want to say that the hidden epistemic assumptions behind scientometrics are those of naïve empiricism, or as it has been described in philosophy of science, of the "received view". ${ }^{15}$ According to this view, scientists are hunting, collecting and representing new for them "facts" rather than intervening into states of affairs by creating models, telling stories, and in turn, interpreting the meanings of those constructed models and the collected data. This is clearly too simplistic an account of science. The problematic

\footnotetext{
${ }^{15}$ See, for instance, (Suppe 1989).
} 
nature of the naïve empiricism has been thoroughly analysed in philosophy of science, and the view has been abandoned decades ago. It would be natural to presuppose that the evaluation procedures are built on the most adequate theoretical account of science; however, in the context of scientometrics, naïve empiricism is still operating. In order to be of value, according to scientometric approach, every assumed discovery and test result must be reported in the form of a scientific publication in a peer-reviewed academic journal. If the results are recognised by the scientific community, citations will soon begin to appear in other journal articles. Both articles and citations can be easily tracked by various research information databases and indexes, making the evaluation on the basis of scientometrics a partially automated procedure. Journals can be evaluated and ranked, enabling publications to be automatically assessed based on their venue. Monographs, however, are more difficult to assess, since they lack the formal indicators which enable a computer to define their category. In Estonia, several attempts have been made to create a list of higher ranking academic publishing houses, to separate them from the "ordinary" or "regular" ones. ${ }^{16}$ Based on publisher rankings, monographs and their sections have been divided into categories similar to journal articles.

Given the dominating formal scientometric evaluation model, we attempt to outline what sort of disciplinary research styles are better suited for getting "high grades", therefore producing higher benefits. On the basis of the descriptions of research cultures, we propose that it is easier for research areas which in Fuchs' terms "produce facts" to fulfil the scientometric requirements. A mutually supportive group, with an effective distribution of labour and low task uncertainty, boasting reliable methodological grounds without any paradigmatic doubts, has good prospects. A certain level of playfulness and readiness to take risks would further improve a research group's prospect for success, as these qualities are valued by fellow researchers, and possibly by the evaluators as well-in case of peerevaluation. This description coincides, to a remarkable extent, with Fuchs' characterisation of cutting-edge laboratory sciences, such as physics in our case study. ${ }^{17}$ However, not all sub-disciplines of physics would necessarily fit in with our proposed model of "guaranteed success". Theoretical physics, a highly prestigious field (like other primarily theoretically groundbreaking sub-disciplines), produces less indisputable "facts", putting researchers in this field at a disadvantage with regard to formal scientometric evaluation.

${ }^{16}$ See (Ross 2012).

${ }^{17}$ It is not surprising that scientometric criteria suit laboratory sciences better, as the underlying epistemic view of empiricism was created on the basis of the simplified image of laboratory science. See, e.g., (Richardson 1998) about early logical empiricism. 
Disciplinary areas are not homogenous in this sense. Theoretical physics retains its high prestige due to its valuable theoretical contribution to the field in spite of the lower position in the automatically generated publication ratings.

As we discussed in section 2, the Thomson Reuters Web of Science simply does not measure the work usually undertaken in the humanities. In the humanities, an internally-valued research outcome is a monograph. To produce such a publication, the individual researcher must have a productive connection with the local culture, and therefore needs time to acquire the knowledge necessary to conceptualise the research object in its wider context. This is where epistemic injustice comes into play: the work done in the humanities tends to be mis-measured, and is therefore discredited as less scientific than other research areas. While the scientometric evaluation criteria suit relatively well to the practices, aims and values of the laboratory sciences, reinforcing their epistemic authority and credibility, the impression created of humanities is poor. At the same time, the research model of the laboratory sciences cannot be adopted in the humanities-since the aims, values, and practices are different, the publication formats accepted in the laboratory sciences cannot be adopted in the humanities. The current evaluation criteria for humanities disciplines in Estonia reflect a sharp value conflict that researchers experience as discrimination:

Ho7: I think behind the rules it's clearly the lack of resources, and argumentation that an article in a well-known journal is an achievement and this gives some people an advantage, and it's worth emphasising it, it's worth making it a rule, and this is what has happened [...] when there are less resources available, and one's research has to go on, it is easiest to suggest that the journal Science is the top of the world, and if you get published there, this is the top of the world, and the rest of the community are not in the top. But it is clear that an historian cannot publish in Science, it's of minimal plausibility, not worth mentioning. [...] [O]ne discipline sets a universalised standard, and thus one gets funding for this, and it works well in the Estonian circumstances.

The interviewed humanities researchers envisage qualitative peer-review as a suitable evaluation model:

Ho2: [In another country] the quality of one's research publications is measured case specifically and qualitatively. [...] And when they are read, the whole picture is looked at, it has a certain complete qualitative measure. And what is looked at first and foremost is whether they have ever written a book. Whether they have written something longer, if they have written a symphony not only a sonata, metaphorically speaking. 
Such a case-specific analysis would demand both significant time and resources. But as long as the humanities are included in such academic research institutions, their evaluation should take the form of a peer-review process, based on expert opinion instead of automatically generated and categorised formal data. To be sure, transparent criteria and shared standards would be required if this model were to be enacted.

For the fair evaluation of academic research in any area, either laboratory science or other, the epistemic assumptions should be placed on adequate basis which should not contradict the internal epistemic aims and values of the disciplines. Scientific research as a narrative enactment is epistemically successful as long as the activities conform to their aims. Thus, evaluation criteria and practices should also conform to a research field's epistemic aims, rather than the needs of bureaucratic information systems. Therefore, the humanities researchers' recommendation to apply more expert assessment for evaluation is compatible with the naturalised philosophy of science perspective: peers in the field share the same kind of aims and values and are prepared to provide adequate evaluations.

\section{Conclusions}

In this paper we compared two academic workplace cultures: the laboratory sciences (physics) and the humanities, with the intention of identifying some cultural characteristics which explain the epistemic injustice in current research evaluation. We borrowed the concept from the work of Alison Wylie (2011) and Miranda Fricker $(1998,2007)$, who discuss what perspectives are underrepresented and undervalued in science, and why. In our work, we took certain disciplinary cultures to be similarly undervalued, due to formal evaluation criteria and practices. As demonstrated by our empirical investigation, researchers in the humanities experience a serious value conflict as they are, for the sake of fundraising, forced to fulfil formal scientometric evaluation criteria by producing easily trackable short-format articles which are endorsed neither by the humanities community nor by wider society. At the same time, the researchers feel that, out of respect for the cultures of their studies, and to contribute to national culture, they should produce thorough monographs. Unfortunately, this format is not well supported by Estonia's official research evaluation standards. Thus, we argue that current academic evaluation standards mis-measure the achievements of the humanities, discrediting their disciplines in comparison to those in the laboratory sciences. In general, contemporary evaluation standards strongly favour the organisational form and outputs of the natural sciences, in which group work dominates and the level of task uncertainty is low. However, alongside time and resource consuming investigation in the humanities, novel and theoretically 
groundbreaking research in the natural sciences also suffers from a credibility deficit in light of these evaluation criteria. For epistemic justice to be restored in research evaluation, the specificity of disciplinary aims, values, and cultural identities must be taken into account.

\section{Acknowledgments}

We would like to thank the reviewer for very detailed and helpful feedback. During the work on this paper, our research was partially funded from the grants ETF7946 and SFo180110so8. The earlier version of the paper was presented at the workshop "Practical Realism-Towards a Realistic Account of Science" organised by the University of Tartu in June 2011; we would like to thank the audience for helpful feedback. Finally, we would like to thank the language editors of our paper, Lisa Dobbin and Marge Paal.

\section{Bibliography}

Adler, N. and Harzing, A. (2009). When knowledge wins: Transcending the sense and nonsense of academic rankings, The Academy of Management Learning and Education 8: 72-95.

Baneyx, A. (2008). "Publish or Perish" as citation metrics used to analyze scientific output in the humanities: International case studies in economics, geography, social sciences, philosophy and history, Archivum Immunologiae et Therapiae Experimentalis 56: 363-371.

Chubin, D. E. and Restivo, S. (1983). The 'mooting' of science studies: Research programmes and science policy, in K. Knorr-Cetina and M. Mulkay (eds), Science Observed: Perspectives on the Social Study of Science, SAGE, London, pp. 53-83.

Fricker, M. (1998). Rational authority and social power. Towards a truly social epistemology, Proceedings of the Aristotelian Society 98: 159-177.

Fricker, M. (2007). Epistemic injustice: Power and the ethics of knowing, Oxford University Press, Oxford.

Fuchs, S. (1992). The Professional Quest for Truth: A Social Theory of Science and Knowledge, State of New York University Press, Albany.

Giere, R. N. (1988). Explaining Science: A Cognitive Approach, University of Chicago Press, Chicago.

Hasse, C. and Trentemøller, S. (2008). Break the Pattern! A critical enquiry into three scientific workplace cultures: Hercules, Caretakers and Worker 
Bees, Tartu University Press, Tartu.

Hasse, C. and Trentemøller, S. (2009). The method of culture contrast, Qualitative Research in Psychology 6: 46-66.

Hasse, C. and Trentemøller, S. (2011). Cultural workplace patterns in academia, Science Studies: An Interdisciplinary Journal for Science and Technology Studies 24: 6-25.

Hemlin, S. and Gustafsson, M. (1996). Research production in the arts and humanities, Scientometrics 37: 417-432.

Longino, H. E. (1990). Science as Social Knowledge. Values and Objectivity in Scientific Inquiry, Princeton University Press, Princeton.

Longino, H. E. (2002). The Fate of Knowledge, Princeton University Press, Princeton.

Richardson, A. W. (1998). Carnap's Construction of the World: The Aufbau and the Emergence of Logical Empiricism, Cambridge University Press, Cambridge.

Rolin, K. (2004). Why gender is a relevant factor in the social epistemology of scientific inquiry, Philosophy of Science 71: 880-891.

Ross, J. (2012). Raamatud, humanitaarteadused ning Eesti teadusbürokraatia, Akadeemia 24: 2265-2271.

Rossiter, M. (1995). Women Scientists in America: Before Affirmative Action, Johns Hopkins University Press, Baltimore.

Rouse, J. (1987). Knowledge and Power: Toward a Political Philosophy of Science, Cornell University Press, Ithaca.

Rouse, J. (1996). Engaging Science: How to Understand its Practices Philosophically, Cornell University Press, Ithaca.

Suppe, F. (1989). The Semantic Conception of Theories and Scientific Realism, University of Illinois Press, Urbana.

Traweek, S. (1992). Border crossings: Narrative strategies in science studies and among physicists in Tsukuba Science City, Japan, in A. Pickering (ed.), Science as Practice and Culture, University of Chicago Press, Chicago, pp. 429-465.

Velbaum, K., Lõhkivi, E. and Tina, M.-L. (2008). UPGEM national report: Estonia, Draw the Line! Universities as workplaces for male and female researchers in Europe, Tartu University Press, Tartu, pp. 137-212.

Wennerås, C. and Wold, A. (2001). Nepotism and sexism in peer-review, in M. Lederman and I. Bartsch (eds), The Gender and Science Reader, Routledge, London, pp. 42-48. 
Wylie, A. (2011). What knowers know well: Women, work and the academy, in H. Grasswick (ed.), Feminist Epistemology and Philosophy of Science: Power in Knowledge, Springer, Dordrecht, pp. 157-179. 